\title{
Modelos de comportamiento ambiental en economía ecológica: Una revisión bibliográfica
}

\author{
JOSÉ MONDÉJAR JIMÉNEZ a , MANUEL VARGAS VARGAS ${ }^{\text {b }}$ \\ a Universidad de Castilla-La Mancha, Facultad de Ciencias Sociales, Avenida de los Alfares, 44, \\ 16071 Cuenca, España.E-mail: jose.mondejar@uclm.es \\ b Universidad de Castilla-La Mancha, Facultad de Ciencias Económicas y Empresariales, Plaza de \\ la Universidad, 1, 02071 Albacete, España.E-mail: manuel.vargas@uclm.es
}

\begin{abstract}
RESUMEN
La economía ecológica, centrada en la utilización, gestión y conservación sostenible de los recursos naturales, renovables o no, supone la inclusión de restricciones ecológicas dentro del marco económico general. Pero el tratamiento de los problemas ambientales presenta un marcado carácter social, y cuya solución implica modificaciones de la conducta personal. Sin embargo, el estudio del comportamiento ambiental individual, sus determinantes y su contrastación empírica sigue siendo un campo de estudio abierto y con consecuencias relevantes para la planificación de las políticas ambientales. Por ello, este trabajo presenta una breve revisión bibliográfica de la evolución de los modelos de comportamiento ambiental, que pueda servir como contextualización de los retos que aún existen en este campo de la economía ecológica.
\end{abstract}

Palabras clave: Comportamiento ambiental; Economía ecológica.

\section{Models of Environmental Behavior in Ecological Economics: A Literature Review}

\begin{abstract}
The ecological economy focused on the use, management, and sustainable conservation of natural resources, renewable or not, implies the inclusion of ecological restrictions within the general economic framework. However, the treatment of environmental problems presents a marked social character, and whose solution implies modifications of personal behavior. Notwithstanding, the study of individual environmental behavior, its determinants, and its empirical testing remain an open field of study with relevant consequences for the planning of environmental policies. Therefore, this work presents a brief bibliographical review of the evolution of environmental behavior models, which can serve as a contextualization of the challenges that still exist in this field of ecological economics.
\end{abstract}

Keywords: Environmental Behavior, Ecological Economics.

Clasificación JEL: Q50, Q57

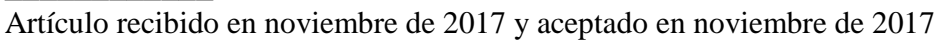

Artículo disponible en versión electrónica en la página www.revista-eea.net, ref. ə-36106 


\section{INTRODUCCIÓN}

En los últimos años se ha incrementado el interés y la preocupación por el impacto de las actividades humanas sobre el medio ambiente y su sostenibilidad, dando lugar a un relativamente nuevo campo de estudio, la economía ecológica. La utilización, gestión y conservación de los recursos naturales, renovables o no, se ha convertido en un foco de análisis multidisciplinar, que imbrica las restricciones ecológicas dentro del marco económico general. La educación, capacitación y la cultura ambiental constituyen unas de las principales herramientas para la gestión económica sostenible del medio ambiente. De hecho, tal como apuntan Zelezny y Schultz (2000), hay un marcado carácter social en el tratamiento de los problemas ambientales, cuya solución implica modificaciones de la conducta individual y social de los individuos. Sin embargo, el estudio del comportamiento ambiental individual, sus determinantes y su contrastación empírica sigue siendo un campo de estudio abierto y con consecuencias relevantes para la planificación de las políticas ambientales.

Por ello, muchos investigadores se han centrado en el estudio de los comportamientos ecológicos o proambientales desde distintas disciplinas científicas y se han acuñado diversos términos como conducta proambiental (Leeming et al., 1993), comportamiento ambiental (Bratt, 1999; Mondéjar et al., 2012), conducta sostenible (Schmuck y Schultz, 2002), conducta ecológica (Kaiser y Shimoda, 1999) o conducta ambiental responsable (Cottrell y Graefe, 1997), con el objetivo de recoger, de forma global, la motivación de los individuos para desarrollar acciones a favor del medio ambiente.

Sin embargo, no existe un acuerdo generalizado sobre las dimensiones que influyen en estas decisiones ni tampoco, a nivel nacional o internacional, se ha consensuado un modelo explicativo del comportamiento ambiental de los individuos que permita planificar la acción de los agentes, públicos o privados, para desarrollar una política eficaz en este campo. Por ello, este trabajo presenta una breve revisión bibliográfica de la evolución de los modelos de comportamiento ambiental, que pueda servir como contextualización de los retos que aún existen en este campo de la economía ecológica.

\section{DESARROLLO SOSTENIBLE Y COMPORTAMIENTO AMBIENTAL}

Tres son las etapas básicas en las que puede estructurarse la evolución histórica del estudio académico sobre el comportamiento ambiental (CorralVerdugo y Queiroz, 2004). Una primera etapa, centrada a finales de los años 60 del siglo XX y en los países industrializados, se inicia con críticas académicas al modelo de crecimiento económico generalizado, basado en la sobreexplotación de los recursos naturales (Dunlap y Van Liere, 1978) y un interés creciente por 
analizar el impacto de las medidas de ahorro de energía y consumo de materias primas sobre la sostenibilidad ambiental (Quesada et al., 2012; Bord y O’Connor, 1997). Paralelamente, desde una óptica conductual, surgen los primeros intentos de conceptualizar el comportamiento ambiental de los individuos, enfocados principalmente hacia el fomento de conductas ambientales responsables (Katzev y Mishima, 1992) o a la denuncia de los efectos de conductas antiecológicas (Agras, Jacob y Ledebeck, 1980).

Es en una segunda etapa, que abarca los dos últimos decenios del siglo pasado, cuando se extiende el análisis del comportamiento ambiental desde una perspectiva interdisciplinaria y sistémica. Sin abandonar los temas energéticos, se amplía el campo de estudio, incluyendo otras facetas como las relacionadas con el reciclaje y la reducción de residuos e incluyendo los efectos de variables demográficas, sociales y económicas (Stern, Dietz y Guagnano, 1995; Guagnano, Stern y Dietz, 1995; Berger, 1997). La conducta ambiental se conceptualiza mediante modelos explicativos, que introducen y fijan las dimensiones básicas del comportamiento individual, como la teoría de la acción razonada (Fishbein y Ajzen, 1975) o el modelo de activación de normas morales (Schwartz, 1977).

Como consecuencia de la generalización del interés por la sostenibilidad ambiental y de la creciente disposición de herramientas conceptuales para su análisis, en el periodo finisecular y los inicios del siglo XXI, aumenta el interés por la gravedad de problemas ambientales, como la escasez de agua (CorralVerdugo, Bechtel y Fraijo-Sing, 2003), el calentamiento global y sus efectos sociales y económicos, como el aumento de la desigualdad social y la pobreza, popularizando el análisis del desarrollo sostenible, no sólo ambiental, sino también social y económico (Scott y Willits, 1994; Mondéjar, Vargas y Mondéjar, 2010; Garau, Mandras y Schirru, 2011; Segarra et al., 2015). Así, el comportamiento ambiental se encuadra como un aspecto básico dentro de un marco más general de sostenibilidad, tanto presente como futura (Schmuck y Schultz, 2002; Hardt y O’Neill, 2017).

Es también el inicio de los grandes planteamientos y acuerdos internacionales para la reducción y reversión de la degradación ambiental, con iniciativas para promover la conservación ambiental (De Young,1996) y acciones políticas concretas (Franzen y Meyer, 2010).

Como consecuencia de esta eclosión de estudios académicos y el creciente interés social y político, Naciones Unidas fomenta una operativización del complejo concepto de desarrollo sostenible que abarca dimensiones económicas, sociales, ambientales e institucionales. Aunque no libre de críticas por el peso concedido a la dimensión económica (Gouveia, 2002), supone el germen de muchas investigaciones y modelos que buscan su refinamiento y adaptación a diversos ámbitos socio-culturales y/o geográficos (Vargas, 2000). 
Sin embargo, los modelos iniciales no abordan la investigación de factores relacionados con las actitudes personales y sociales subyacentes. Aunque establecen los aspectos ambientales básicos vinculados al desarrollo sostenible, no analizan cuáles son y cómo se generan y extienden las acciones individuales que inciden en dicho ámbito. Se dificulta así abordar la aparente paradoja de la disociación entre el incremento notable de la conciencia ecológica y el desarrollo de actitudes y comportamientos sostenibles individuales (Stern Dietz y Guagnano, 1995).

\section{MODELOS DE COMPORTAMIENTO AMBIENTAL SOSTENIBLE}

Dentro del ámbito individual del comportamiento ambiental, uno de los primeros enfoques se centró en el estudio de las preferencias ambientales manifestadas (Carlsson y Johansson, 2000; Popp, 2001; Bulte et al., 2005), del efecto de varios factores sociales, demográficos y económicos (Engel y Pötschke, 1998; Israel y Levinson, 2004; Torgler y García, 2007) o del impacto de las creencias ambientales sobre consumo, reciclaje o ahorro de recursos (CorralVerdugo, Bechtel y Fraijo-Sing, 2003; Karp, 1996). También se ha relacionado el comportamiento ambiental con factores como el nivel de riqueza (Franzen 2003), el comportamiento de productores y consumidores (Franzen y Meyer 2010), o las pautas de consumo sostenible (Mainieri et al., 1997; Ebreo, Hershey y Vinning 1999).

El marco teórico generalmente adoptado para el estudio del comportamiento ambiental individual es el de valor, normas y creencias hacia el medio ambiente (Stern 2000; Martins, 2018), que agrupa los factores determinantes en actitudinales, sociales, individuales y de hábitos automatizados. Centrándose en el primer grupo de factores, los trabajos empíricos no han sido concluyentes (Steel,1996; Iwata, 2001), mostrando que una actitud proambiental puede coexistir con la ausencia de comportamientos responsables (Scott y Willits,1994; Kaiser, Wolfing y Fuhrer 1999). Diversos trabajos han abordado esta dicotomía optando por la inclusión de variables relacionadas con valores, comportamiento y creencias (Kaiser et al., 1999; Stern et al., 1999; Pérez et al., 2011; Nordlund y Garvill, 2002; Garling et al., 2003).

\section{CONCLUSIONES}

A pesar del auge de enfoques como la economía sostenible o la economía ecológica, el grueso de la investigación se ha centrado en aspectos macroeconómicos (Daly, 1991) o en el análisis de la política económica con restricciones de sostenibilidad ambiental (Dopico e Iglesias, 2010). Los modelos individuales de comportamiento ambiental, sin embargo, siguen siendo un campo de estudio abierto, tanto por el limitado éxito de los trabajos existentes 
(Berenguer y Corraliza, 2000; Berenguer et al., 2000) como por su integración dentro del marco general de análisis que permita un desarrollo económico sostenible, tanto ambiental como socialmente. Este trabajo presenta un somero repaso de los modelos desarrollados en los últimos treinta años, que pueda servir como punto de partida para abordar los retos actuales de comprender y modelizar los comportamientos ambientales individuales, factor relevante para la sostenibilidad y que ha presentado un menor desarrollo en la construcción y eficacia de las medidas de política ambiental adoptadas.

\section{REFERENCIAS BIBLIOGRÁFICAS}

AGRAS, W.S.; JACOB, R.G. y LEDEBECK, M. (1980). "The California drought: A quasi experimental analysis of social policy". Journal of Applied Behavior Analysis, 13, pp. 561-570.

BERENGUER, J.M. y CORRALIZA, J.A. (2000). "Preocupación ambiental y comportamientos ecológicos". Psicothema, 12(3), pp. 325-329.

BERENGUER, J.M.; CORRALIZA, J.A.; MARTíN, R. y OCEJA, L. (2000). "Preocupación ecológica y acciones ambientales. Un proceso interactivo". Estudios de Psicología, 22(1), pp. 37-52.

BERGER, I. (1997). "The demographics of recycling and the structure of environmental behavior". Environment and Behavior, 29, pp. 515-531.

BORD, R.J. y O'CONNOR, R.E. (1997). "The gender gap in environmental attitudes: the case of perceived vulnerability to risk". Social Science Quarterly, 78, pp. 830-840.

BRATT, C. (1999). "Consumers' environmental behavior: Generalized, sector-based, or compensatory?". Environment and Behavior, 31, pp. 28-44.

BULTE, E.; GERKING, S.; LIST, J.A. y DE ZEEUW, A. (2005). "The effect of varying the causes of environmental problems on stated WTP values: evidence from a field study". Journal of Environmental Economics and Management, 49, pp. 330-342.

CARLSSON, F. y JOHANSSON, O. (2000). "Willingness to pay for improved air quality in Sweden". Applied Economics, 32, pp. 661-669.

CORRAL-VERDUGO, V.; BECHTEL, R.B. y FRAIJO-SING, B. (2003). "Environmental beliefs and water conservation: An empirical study". Journal of Environmental Psychology, 23, pp. 247-257.

CORRAL-VERDUGO, V. y QUEIROZ, J. (2004). "Aproximaciones al estudio de la conducta sustentable". Medio Ambiente y Comportamiento Humano, 5(1-2), pp. 1-26.

COTTRELL, S.P. y GRAEFE, A.R. (1997). "Testing a conceptual framework of responsable environmental behavior". Journal of Environmental Education, 29, pp. 1727.

DALY, H. (1991). "Towards an environmental macroeconomics". Land Economics, 67(2), pp. 255-259.

DE YOUNG, R. (1996). "Some psychological aspects of reduced consumption behavior. The role of intrinsic motivation and competence motivation". Environment and Behavior, 28, pp. 358-409. 
DOPICO, J.A. e IGLESIAS, G. (2010). Economía sostenible. Teoría y política. La Coruña: Netbiblo.

DUNLAP, R.E. y VAN LIERE, K.D. (1978). "The New Environmental Paradigm. A proposed measuring and preliminary results". Journal of Environmental Education, 9, pp. 10-19.

EBREO, A.; HERSHEY, J. y VINING, J. (1999). "Reducing solid waste: Linking recycling to environmentally responsible consumerism". Environment and Behavior, 31, pp. 107135.

FISHBEIN, M. y AJZEN, I. (1975). Belief, attitude, intention and behavior: an introduction to theory and research. Reading, MA: Addison-Wesley.

FRANZEN, A. (2003). "Environmental Attitudes in International Comparison: An Analysis of the ISSP Surveys 1993 and 2000". Social Science Quarterly, 84, pp. 297-308.

FRANZEN, A. y MEYER, R. (2010). "Environmental Attitudes in Cross-National Perspective: A Multilevel Analysis of the ISSP 1993 and 2000". European Sociological Review, 26(2), pp. 219-234.

GARAU, G.; MANDRAS, G. y SCHIRRU, L. (2011). "A statistical information system supporting environmental policies". Environmental Engineering and Management Journal, 10(12), pp. 1903-1910.

GÄRLING, T.; FUJII, S.; GÄRLING, A. y JAKOBSSON, C. (2003). "Moderating effects of social value orientation on determinants of proenvironmental behavior intention". Journal of Environmental Psychology, 23, pp. 1-3.

GOUVEIA, V.V. (2002). "Self, culture, and sustainable development". En P. Schmuck y P.W. Schultz (eds.): Psychology of Sustainable Development (pp. 151-174). Boston, MA: Springer.

GUAGNANO, G.A.; STERN, P.C. y DIETZ, T. (1995). "Influences on attitude-behavior relationships: A natural experiment with curbside recycling". Environment and Behavior, 27, pp. 699-718.

HARDT, L. y O'NEILL, D.W. (2017). "Ecological Macroeconomic Models: Assessing Current Developments". Ecological Economics, 134, pp. 198-211.

ISRAEL, D. y LEVINSON, A. (2004). "Willingness to pay for environmental quality: testable empirical implications of the growth and environmental literature". Contributions to Economic Analysis and Policy, 3(1), pp. 1-31.

IWATA, O. (2001). "Attitudinal determinants of environmentally responsible behavior". Social Behavior and Personality, 29, pp. 183-190.

KAISER, F.G.; RANNEY, M.; HARTIG, T.; y BOWLER, P.A. (1999). "Ecological behaviour, environmental attitude and feelings of responsibility for the environment". European Psychologist, 4(2), pp. 59-74.

KAISER, F.G. y SHIMODA, T.A. (1999). "Responsibility as a predictor of ecological behavior". Journal of Environmental Psychology, 19, pp. 243-253.

KAISER, F.G.; WÖLFING, S. y FUHRER, U. (1999). "Environmental attitude and ecological behavior". Journal of Environmental Psychology, 19(1), pp. 1-19.

KARP, D.G. (1996). "Values and their effect on pro-environmental behavior". Environment and Behavior, 28(1), pp. 111-133.

KATZEV, R. y MISHIMA, H.R. (1992). "The use of posted feedback to promote recycling". Psychological Reports, 71, pp. 259-264. 
LEEMING, F.; DWYER, W.; PORTER, B. y COBERN, M. (1993). "Outcome research in environmental education: A critical review". Journal of Environmental Education, 24, pp. 8-21.

MAINIERI, T.; BARNETT, E.; VALDERO, T.; UNIPAN, J. y OSKAMP, S. (1997). "Green buying: The influence of environmental concern on consumer behavior". The Journal of Social Psychology, 13, pp. 189-204.

MARTINS, N.O. (2018). "The Classical Circular Economy, Sraffian Ecological Economics and the Capabilities Approach". Ecological Economics, 145, pp. 38-45.

MONDÉJAR, J.; MONDÉJAR, J.A; VARGAS, M. y GÁZQUEZ, J.C (2012). "Personal attitudes in environmental protection". International Journal of Environmental Research, 6(4), pp. 1039-1044.

MONDÉJAR, J.; VARGAS, M. y MONDÉJAR, J.A. (2010). "Measuring environmental evolution using synthetic indicators". Environmental Engineering and Management Journal, 9(9), pp. 1145-1149.

NORDLUND, A.M. y GARVILL, J. (2002). "Value structures behind proenvironmental behavior". Environment and Behavior, 34(6), pp. 740-756.

PÉREZ, E.; MILANÉS, P.; MESEGUER, M.L. y MONDÉJAR, J. (2011). "Eco-efficiency: Effects on economic and financial performance. Evidences from Dow Jones sustainability Europe index". Environmental Engineering and Management Journal, 10(12), pp. 1801-1808

POPP, D. (2001). "Altruism and the demand for environmental quality". Land Economics, 77(3), pp. 339-349.

QUESADA, J.M.; VILLAR, E.; MONDÉJAR, J. y MOLINA, V. (2011). "Carbon dioxide emissions vs. allocation rights: Spanish case analysis". International Journal of Environmental Research, 5(2), pp. 469-474

SCHMUCK, P. y SCHULTZ, P.W. (2002). "Sustainable development as a challenge for Psychology". En P. Schmuck y P.W. Schultz (eds.): Psychology of Sustainable Development (pp. 3-17). Boston, MA: Springer.

SCHWARTZ, S.H. (1977). "Normative influences on altruism". En L. Berkowitz (ed.): Advances in experimental social psychology (pp. 221-279). Nueva York: Academic Press.

SCOTT, D. y WILLITS, F.K. (1994). "Environmental attitudes and behavior. A Pennsylvania survey". Environment and Behavior, 26(2), pp. 239-260

SEGARRA, M.; MONDÉJAR, J.; PEIRÓ, A. y MONDÉJAR, J.A. (2015). "Heterogeneous behavioral patterns influencing the proactive environmental orientation of firms: How does your company look?". Innovation: Management, Policy \& Practice, 17(1), pp. 6980.

STEEL, B.S. (1996). "Thinking globally and acting locally: environmental attitudes, behavior and activism". Journal of Environmental Management, 47, pp. 27-36.

STERN, P.C. (2000). "Toward a coherent theory of environmentally significant behaviour". Journal of Social Issues, 56(3), pp. 407-424.

STERN, P.C.; DIETZ, T. y GUAGNANO, G.A. (1995). "The New Ecological Paradigm in social-psychological context". Environment and Behavior, 27(6), pp. 723-743.

TORGLER, B. y GARCIA, M.A. (2007). "The determinants of individuals' attitudes towards preventing environmental damage". Ecological Economics, 63(2-3), pp. 536-552.

VARGAS, C.M. (2000). "Sustainable development education: Averting or mitigating cultural collision". International Journal of Educational Development, 20, pp. 377-396. 
ZELEZNY, L.C. y SCHULTZ, P.W. (2000). "Promoting Environmentalism". Journal of Social Issues, 56(3), pp. 365-371. 